\title{
Glycogen storage disease due to muscle glycogen phosphorylase deficiency
}

INSERM

\section{Source}

INSERM. (1999). Orphanet: an online rare disease and orphan drug data base. Glycogen storage disease due to muscle glycogen phosphorylase deficiency. ORPHA:368

Myophosphorylase deficiency (McArdle's disease), or glycogen storage disease type 5 (GSD5), is a severe form of glycogen storage disease characterized by exercise intolerance. 